\title{
A Gd-Based Glucosamine Magnetic Resonance Imaging (MRI) Contrast Agent That Crosses the Intact Blood-Brain-Barrier for Entry into Cortical Neurons.
}

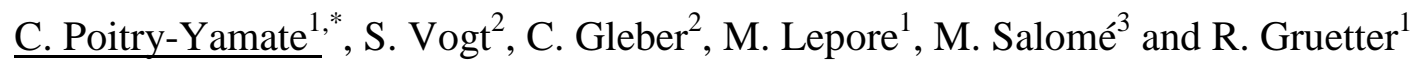 \\ 1. Centre d'Imagerie Biomédicale, Ecole Polytechnique Fédérale de Lausanne, Lausanne, Switzerland \\ 2. Advanced Photon Source, Argonne National Laboratory, Chicago, Ill. USA \\ 3. European Synchrotron Radiation Facility, Grenoble, France \\ * Corresponding author, carole.poitry-yamate@epfl.ch
}

Introduction. Chelated gadolinium, e.g. Gd-DTPA [1] is an extracellular (para)magnetic resonance imaging (MRI) contrast agent with widespread clinical use, e.g. brain, to assess disruption of the blood brain barrier (BBB). A cell permeable form, Gd-DTPA-glucosamine (Gd-DTPA-GlcN), was recently developed for imaging peripheral tumors [2], but its functionality remains untested for the brain. This is unfortunate since GlcN, the delivery vehicle moiety, is a naturally occurring sugar in a branch of glycolysis with features in common with 2-deoxy-D-glucose (DG), the conventional glucose analogue: (1) both sugars enter cells on GLUT transporter proteins [3] found on neurons and astrocytes; and (2) the cellular metabolism of both sugars is essentially limited to their phosphorylation by hexokinase, enabling their intracellular trapping [4]. In MRI, gadolinium is not itself directly imaged. For this reason, we set out to validate Gd-DTPA-GlcN for brain imaging: Gd-DTPA-GlcN was administered at a clinical dose $(\sim 0.3 \mathrm{mmol} / \mathrm{kg})$ to the healthy male rat. Thereafter, $\mathrm{x}$-ray fluorescence chemical imaging of such brains, albeit freeze-substitution-fixed [5] and epon infiltrated, enabled assessing probe functionality: does it cross the BBB? Is it transported into brain, and retained intracellularly in situ?

Motivation. Using a fluorescence microscope operating at $7.33 \mathrm{keV}$ and an unfocused 100 micron monochromatic beam for punctual measurements at ESRF beamline ID21, we sought spectral information of the probe's function, with and without GlcN, from the inner surface of lyophilized rat retinae. Metabolically active (dark-adapted) retinae were initially superfused with physiological saline solution carrying either Gd-DTPA-GlcN or Gd-DTPA $(500 \mu \mathrm{M}$ each). A 50 min superfusion with GdDTPA-GlcN resulted in a robust $\mathrm{Gd} \mathrm{L}_{\alpha 1}$ fluorescence photon peak at $6 \mathrm{keV}$ (Fig. 1a, left), confirmed by Gd-DTPA-GlcN standards (Fig. 1a, right, inset). Superfusion with Gd-DTPA resulted in a significantly lower Gd fluorescence emission signal, likely extracellular in origin. We concluded that the chelated gadolinium must obligatorily be linked to GlcN for intracellular uptake and retention (by trapping) in the light-sensitive portion of the central nervous system (CNS).

Methods The aforementioned questions were addressed by performing 2-dimensional distribution mapping of Gd at APS beamline 2-ID-E. Mapping P and S in parallel at 500 $\mathrm{nm}^{2}$ spatial resolution from $6 \mu \mathrm{m}$ thick brain slices placed the metabolic maps in a cell-type context. An incident monochromatic 10 $\mathrm{keV}$ x-ray beam focused to a $\sim 0.5$ micron diameter spot was used. Samples were raster scanned with respect to the incident beam. Spectra were collected for $250 \mathrm{~ms}$ or $25 \mathrm{~ms}$ per pixel (fly scans) or $2 \mathrm{~s}$ per pixel (step scans) using an energy-dispersive Ge detector. Fitting and background corrected spectra were obtained with reference to National Institute of Standard and Technology thin film standards. Panels of Figure $1 \mathrm{~b}$ and $\mathrm{c}$ were constructed using MAPS software [6]. 
Main results. Over a widespread area of brain cortex (Fig. 1, Panel b) Gd content (3.0-25.0 $\mu \mathrm{g} / \mathrm{cm}^{2}$ ) was confined to the cytoplasm of pyramidal neurons. Cell soma $S$ content $\left(2.0\right.$ to $\left.4.55 \mu \mathrm{g} / \mathrm{cm}^{2}\right)$ was cytosolic, and outside the cell nucleus. $\mathrm{Cu}\left(0.006-0.0170 \mu \mathrm{g} / \mathrm{cm}^{2}\right)$ and $\mathrm{Zn}\left(0.20-3.23 \mu \mathrm{g} / \mathrm{cm}^{2}\right)$ were also discernible in the neuronal cytoplasm. Ca content $\left(0.025-0.180 \mu \mathrm{g} / \mathrm{cm}^{2}\right)$ was highest in a small population of neurons. Line plots of Gd content, from low to high were: the epon matrix (left half of dotted region in the Gd map), cell nucleus, neuropil and cytosol. At the single cell level (Panel c, area in Panel b), Gd (0.0087 to $\left.0.0095 \mu \mathrm{g} / \mathrm{cm}^{2}\right)$ content was cytosolic in neurons (*) and glia (arrow).

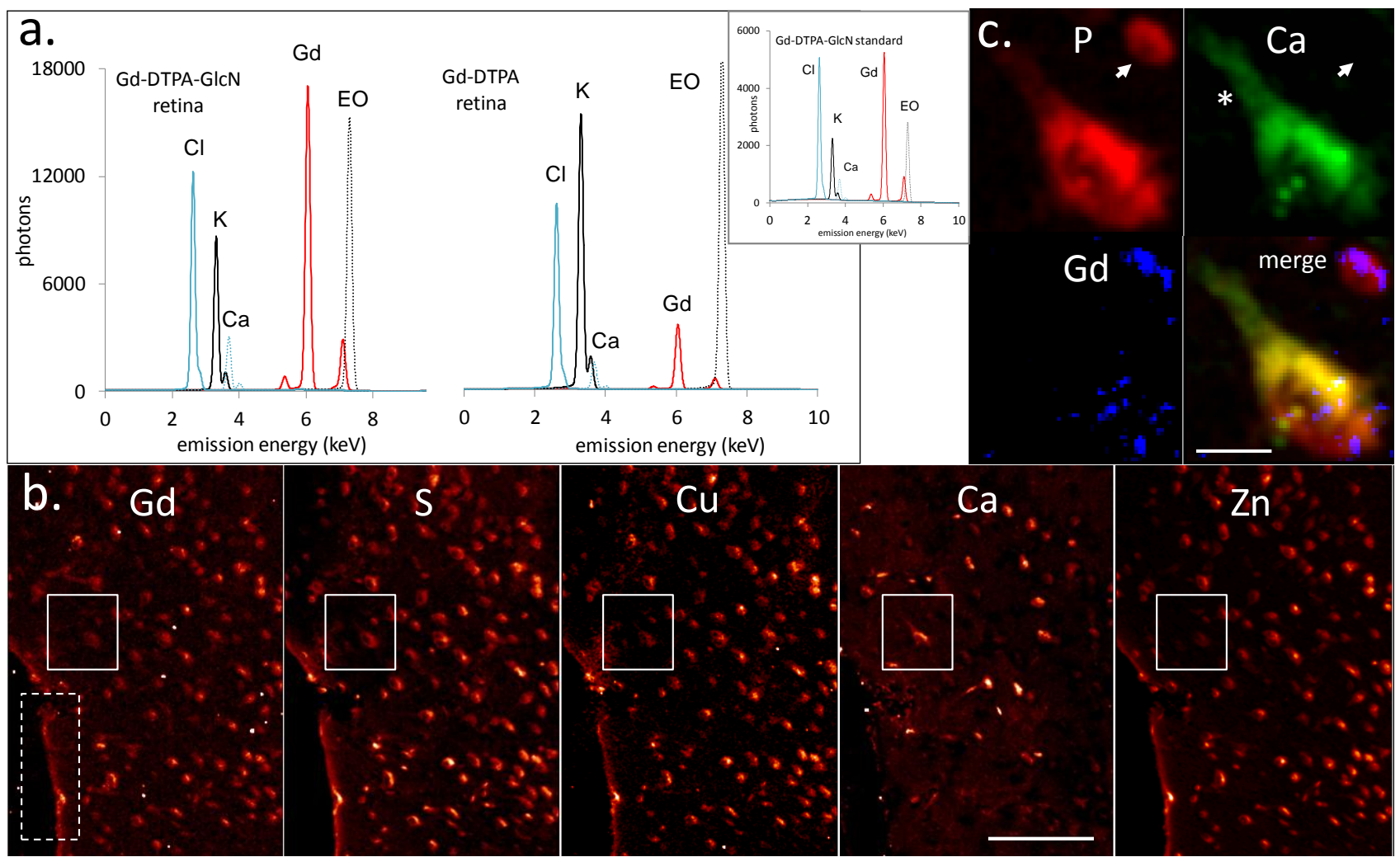

Figure 1. Functionality of Gd-DTPA-GIcN in mammalian CNS. (a) spectra from retinae, previously superfused with the complete or incomplete probe. Inset, Gd-DTPA-GlcN standard. (b) chemical fluorescence maps of $\mathrm{Gd}, \mathrm{S}, \mathrm{Cu}, \mathrm{Ca}$ and $\mathrm{Zn}$ in rat cortex, 50 min following delivery of Gd-DTPA-GlcN in the healthy rat. Scale: $100 \mu \mathrm{m}$. (c) Probe retention in single cells from region in b. Scale: $10 \mu \mathrm{m}$.

Conclusion. Following its intravenous delivery, Gd-DTPA-GlcN was taken up by cortical neurons in vivo (Fig.1b); by inference, the probe physically crossed the BBB for entry into the brain. Neurons retained Gd, presumably as trapped Gd-DTPA-GlcN-6P. Although Zn and $\mathrm{Cu}$ transmetallation cannot be excluded [7], the strategy implemented here forms a useful basis for understanding the cellular contribution to in vivo MRI imaging of brain and tumors using GlcN, a new CEST MRI agent [3].

\section{References}

[1] Weinmann et al. (1984) AJR 142:619-624.

[2] Zhang et al. (2011) European J Radiol. 79:369-374.

[3] Rivlin \& Navon (2016) Nature Scientific Reports. DOI:10.1038/srep32648.

[4] Poitry-Yamate et al (2012) JNR, DOI:10.1002/jnr.23171. 
[5] Yarowsky \& Boyne (1989) J Neurocytol. 18:121-135.

[6] Vogt S. see, https://www.aps.anl.gov/SXSPM/Other-Projects/Fluorescence-Analysis

[7] Perazella (2009) Clin J Am Soc Nephrol 4:461-469.

[8] The authors gratefully acknowledge Dr. A-S Chauvin for the probe; CIBM-PET core funding; and the ESRF and APS for provision of beam time. 
https://doi.org/10.1017/S1431927618014186 Published online by Cambridge University Press 